\section{Severe case of intestinal vasculitis: knife's edge diagnosis and treatment}

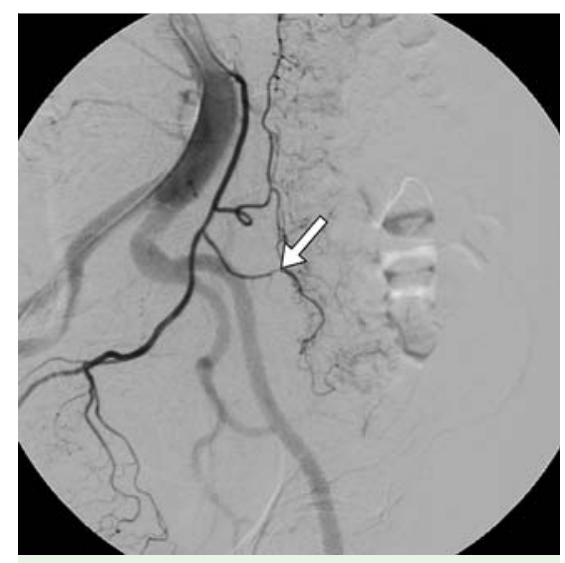

Fig. 1 Abdominal angiogram in a 75-year-old Caucasian woman with severe enteritis of unknown origin showing luminal irregularity in multiple visceral branches of the inferior mesenteric artery typical for intestinal vasculitis (white arrow)

A 75-year-old Caucasian woman was referred to our department due to severe enteritis of unknown origin. Peroral gastrointestinal endoscopy with a long enteroscope (SIF-Q180, Olympus Optical, Tokyo, Japan) revealed severe, segmented, partially stenotic, ulcerative duodenitis/ jejunitis ( Video 1 ). Peranal single-balloon enteroscopy $(210 \mathrm{~cm})$ showed no pathologic processes, and cytomegalovirus infection [1] and nonsteroidal antiinflammatory drug (NSAID)-induced enteropathy [2] were ruled out. Digital subtraction angiography was carried out to detect any ischemic cause of the intestinal alterations. Irregularities were noted

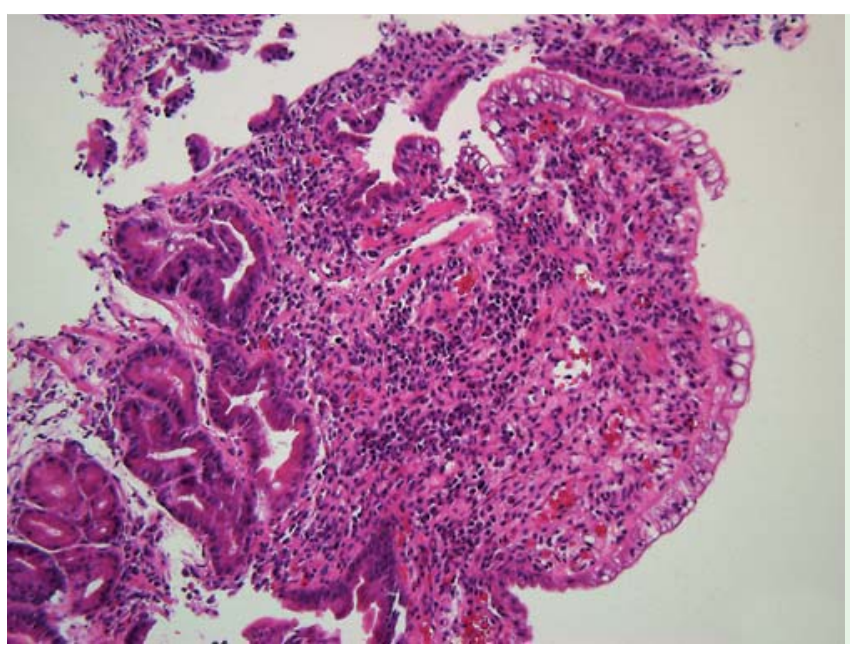

in the caliber of the superior mesenteric artery and inferior mesenteric artery ( $\bullet$ Fig. 1), both in the central and peripheral branches, typical for intestinal vasculitis. The diagnosis was confirmed by histological examination ( $\bullet$ Fig. 2 ), and immunosuppressive treatment with steroid and azathioprine was instituted. Initially, the patient responded well, but on follow-up the intestinal vasculitis showed only slight macroscopic improvement. At 6 months after the initial diagnosis, the patient was referred to our neurological department with dysphasia and suspected cerebral infarction. Magnetic resonance angiography revealed ischemic regions, probably because of cerebral involvement in the systemic vasculitis [3]. The treatment was changed to cyclophosphamide and the patient recovered well without any neurological sequelae. At the latest (12-month) follow-up, the intestinal vasculitis was controlled with the cyclophosphamide treatment, with a satisfactory clinical outcome, although mucosal alterations persisted endoscopically. Vasculitis is a rare disease entity. The diagnosis is complex and has to be confirmed using a multimodal approach. In addition, the clinical manifestations of

\section{Video 1}

Upper gastrointestinal endoscopy showing severe, segmented, partially stenotic, ulcerative duodenitis/jejunitis. systemic vasculitides are often restricted to distinct parts of the human body, such as the intestine, resulting in symptoms that are not easy to interpret. Patients are often first seen by gastroenterologists because the most common symptoms are abdominal pain and gastrointestinal bleeding, as in the reported patient. The present report highlights the need for physicians to be aware of intestinal vasculitis, especially in patients with unusual endoscopic findings $[4,5]$.

\section{Endoscopy_UCTN_Code_CCL_1AC_2AB}

\section{Competing interests: None}

\section{P. Lenz'1 , J. Dominick¹, P. J. Barth², M. Köhler ${ }^{3}$, D. Domagk ${ }^{1}$, K. Hengst ${ }^{1}$, H. Ullerich ${ }^{1}$}

${ }^{1}$ Department of Medicine B, University of Muenster, Muenster, Germany

${ }^{2}$ Gerhard-Domagk-Institute of Pathology, University of Muenster, Muenster, Germany

${ }^{3}$ Department of Clinical Radiology, University of Muenster, Muenster, Germany

\section{References}

1 Cheung AN, $\mathrm{Ng} I O$. Cytomegalovirus infection of the gastrointestinal tract in non-AIDS patients. Am J Gastroenterol 1993; 88: 1882 1886

2 Higuchi K, Umegaki E, Watanabe T et al. Present status and strategy of NSAIDs-induced small bowel injury. J Gastroenterol 2009; 44: 879-888

3 Minagar A, Fowler M, Harris MK et al. Neurologic presentations of systemic vasculitides. Neurol Clin 2010; 28: 171 - 184

4 Morgan MD, Savage CO. Vasculitis in the gastrointestinal tract. Best Pract Res Clin Gastroenterol 2005; 19: 215-233

5 Muller-Ladner U. Vasculitides of the gastrointestinal tract. Best Pract Res Clin Gastroenterol 2001; 15: 59-82

Fig. 2 Histological section showing mucosal architectural distortions, stromal fibrosis, and epithelial degeneration consistent with small-bowel ischemia. The small vessels were normal, and no features of vasculitis or thrombosis were seen.

\section{Bibliography}

Dol http://dx.doi.org/

10.1055/s-0032-1306789

Endoscopy 2012; 44: E128

(c) Georg Thieme Verlag KG

Stuttgart · New York

ISSN 0013-726X

\author{
Corresponding author \\ P. Lenz \\ Department of Medicine B \\ University of Muenster \\ Albert-Schweitzer-Campus 1, Building A1 \\ D-48149 Muenster \\ Germany \\ lenz.philipp@ukmuenster.de
}

